\title{
Higher overcommitment to work is associated with higher plasma cortisol but not ACTH responses in the combined dexamethasone/CRH test in apparently healthy men and women
}

\author{
Petra H. Wirtz ${ }^{a}$, Johannes Siegrist ${ }^{b}$, Anna Schuhmacher ${ }^{c}$, Susanne Hoefels ${ }^{c}$, \\ Wolfgang Maier ${ }^{c}$, Astrid W. Zobel ${ }^{c, *}$ \\ ${ }^{a}$ Department of Clinical Psychology and Psychotherapy, University of Zurich, Switzerland \\ ${ }^{\mathrm{b}}$ Department of Medical Sociology, University of Duesseldorf, Germany \\ ${ }^{\mathrm{c}}$ Department of Psychiatry, University of Bonn, Sigmund-Freud-Strasse 25, 53105 Bonn, Germany
}

\author{
KEYWORDS \\ Overcommitment; \\ Combined \\ dexamethasone/ \\ $\mathrm{CRH}$ test; \\ Cortisol; \\ ACTH; \\ Cardiovascular disease \\ risk; \\ Depressive symptoms
}

\begin{abstract}
Summary
Background: Overcommitment $(\mathrm{OC})$ is a pattern of excessive striving that has been associated with alterations in the hypothalamus-pituitary-adrenal (HPA) system. To investigate whether overcommitment is associated with alterations in HPA system function we measured cortisol and adrenocorticotropin (ACTH) release in response to the combined dexamethasone/CRH test. Methods: We recruited 92 men and 108 women of a wide range of OC scores including the minimum (6) and maximum (24) of possible OC scores (mean \pm SEM: $13.25 \pm .27$ ). We repeatedly measured plasma cortisol and ACTH levels in the combined dexamethasone/CRH test after injection of $100 \mu \mathrm{l} \mathrm{CRH}$ preceded by administration of $1.5 \mathrm{mg}$ dexamethasone the night before. Moreover, we assessed depressive symptoms (Beck Depression Inventory, BDI) and work stress (effort-reward-imbalance, ERI).

Results: Independent of age and gender, higher $\mathrm{OC}$ was associated with higher repeated cortisol (interaction time-by-OC: $p=.014, f=.15)$ but not ACTH $(p=.22)$ secretion in the combined dexamethasone/CRH test. Similarly, higher cortisol $\left(\beta=.16, p=.029, R^{2}=.02\right)$ but not ACTH $(p=.47)$ increase following CRH injection was predicted by higher OC. Depressive symptoms (BDI score) and work stress scores (effort-reward-ratio) did not relate to neuroendocrine responses to the dexamethasone/CRH test. Controlling for depressive symptoms and work stress scores in addition to age and gender did not change results. OC was not associated with ACTH or cortisol pre-test levels.
\end{abstract}

\footnotetext{
* Corresponding author. Tel.: +49 228287 15717; fax: +49228 28716097.

E-mail address: Astrid.Zobel@ukb.uni-bonn.de (A.W. Zobel).
} 
Discussion: Whereas $\mathrm{OC}$ was not associated with alterations in negative feedback sensitivity after dexamethasone administration, our findings indicate that with increasing OC scores, a higher reactivity of the adrenal cortex together with a normal reactivity of the pituitary is observed following subsequent stimulation by $\mathrm{CRH}$ injection.

\section{Introduction}

Overcommitment $(\mathrm{OC})$ is an enduring cognitive-motivational pattern of maladaptive coping with demands characterized by the inability to withdraw from obligations combined with a high need for control and approval (Siegrist et al., 2004). Overcommitted individuals are extremely ambitious and tend to repeatedly exaggerate their efforts while at the same time overtaxing their resources. This consequently precipitates exhaustion and poor health (Joksimovic et al., 1999). OC has been introduced as an intrinsic component to the model of effort-reward imbalance (ERI) at work. In this context, $\mathrm{OC}$ is thought to magnify stressful experience resulting from high cost/low gain conditions at work because it induces exaggerated efforts which are not met by extrinsic rewards (Siegrist, 1996).

Several prospective studies suggest that OC independently increases coronary heart disease (CHD) risk (Siegrist et al., 1990; Joksimovic et al., 1999; Kuper et al., 2002) and the underlying biological pathways are beginning to be understood. Studies assessing hypothalamus-pituitaryadrenal (HPA) axis activity and reactivity suggest a potential role for alterations in the HPA system with OC. Cortisol levels over the work day were shown to be elevated with OC (Hurwitz-Eller et al., 2006). Similarly, the increase in cortisol over the 30 min after awakening was found to be greater in overcommitted men (Steptoe et al., 2004). With respect to stress reactivity, we and others recently demonstrated associations between OC and blunted HPA axis stress reactivity. Upon acute psychosocial stress stimulation, higher $\mathrm{OC}$ was associated with lower salivary and plasma cortisol as well as adenocorticotropin (ACTH) levels in reaction to the stressor (Bellingrath and Kudielka, 2008; Wirtz et al., 2008).

So far, little is known about associations between $O C$ and alterations in HPA system function. One recent study assessed HPA system function by means of the dexamethasone suppression test (DST) and did not report associations between OC and salivary cortisol secretion after pre-medication with $.25 \mathrm{mg}$ of dexamethasone (Bellingrath et al., 2008). Notably, lack of sensitivity of the DST has been frequently criticized (Arana et al., 1985; Ising et al., 2005), and in order to overcome this limitation, a combined dexamethasone/corticotropin-releasing hormone (dex/CRH) test was proposed by Holsboer et al. (1987). This test not only reflects the suppressive effects of a dexamethasone dose administered the day before but also reflects the additional effects of a $\mathrm{CRH}$ injection on subsequent pituitary and adrenal cortex reactivity. To date, this test is considered the most sensitive tool for detection of alterations in HPA system function (Heuser et al., 1994). Morover, it is refractory against confounding influences such as caffeine and nicotine consumption, body weight, and acute stressors during the test (Kunzel et al., 2003).

The combined dex/CRH test has been frequently used in researching an independent psychological risk factor for $\mathrm{CHD}$ other than OC, namely depression (Musselman et al., 1998; Zobel et al., 2001; Lett et al., 2004; Ising et al., 2005; Parissis et al., 2007; Van der Kooy et al., 2007). Results of the combined dex/CRH test demonstrate that depression and depressive symptoms are associated with elevations in hormonal responses, particularly of cortisol (Zobel et al., 2001; Hatzinger et al., 2002; Ising et al., 2005). Elevated hormonal responses to the dex/CRH test in depression have been proposed to reflect impaired glucocorticoid functioning, a key mechanism in the pathogenesis of depression (Ising et al., 2005) that might at least in part underlie the increased CHD risk with depression (Girod and Brotman, 2004; Lett et al., 2004; Parissis et al., 2007). However, to date it is not known whether elevated hormonal responses to the dex/CRH test as observed in depression can be observed in other independent psychological risk factors for CHD such as OC.

We therefore set out to investigate whether $O C$ is associated with alterations in HPA system function in a sizable number of healthy men and women. We assessed $O C$ and repeatedly measured cortisol and $\mathrm{ACTH}$ plasma levels in the combined dexamethasone/CRH test. Moreover, we assessed depressive symptoms and work stress as measured by the imbalance between effort and reward as potential confounders. We hypothesized that similar to observations regarding depressive symptoms, higher OC scores would be associated with higher cortisol release following $\mathrm{CRH}$ injection.

\section{Materials and methods}

\section{Subjects}

The final sample size consisted of 200 persons with complete OC scores as well as cortisol and ACTH responses to $\mathrm{CRH}$ infusion. After a complete description of the procedures to the subjects, written informed consent was obtained. The Ethics Committee of the University of Bonn formally approved the research protocol. We intentionally recruited non-smoking medication-free men and women of a wide $\mathrm{OC}$ range including all possible OC scores (6-24) who were in excellent physical and mental health. The volunteers were identified through the register of residents in Bonn: letters containing information about the purpose and the procedure of the study as well as the request for participation for financial allowance in case of full physical and mental health were sent to 900 subjects. Due to local data protection regulations we have no access to information on the non-participating subjects.

A thorough medical examination was performed in order to record general medical conditions, psychiatric and medical disorders, lifestyle, psychosocial problems, and stress. Subjects with severe or chronic diseases or subjects taking any medication besides oral contraceptives were excluded from the study. Specific exclusion criteria were: psychiatric disorders including major depression, regular strenuous exercise, alcohol and illicit drug abuse; any heart disease, varicosis or 
thrombotic diseases, elevated blood sugar and diabetes, elevated cholesterol, liver and renal diseases, chronic obstructive pulmonary disease, allergies and atopic diathesis, rheumatic diseases, and current infectious diseases. In order to screen for the presence of psychopathological symptoms the DIA-X-SSQ questionnaire (Wittchen and Perkonigg, 1997) was administered. This comprehensive clinical interview instrument detects psychiatric disorders with high sensitivity (86\%) (Wittchen and Pfister, 1997). Of the initially recruited probands $17.3 \%$ had to be excluded because they fulfilled the criteria for psychiatric disorders (50\% because of affective disorders). Of the further probands $8.4 \%$ had to be excluded as they had been treated for mental disorders in the past.

\section{Psychological assessment}

Overcommitment was assessed by a uni-dimensional scale composed by 6 Likert-scaled items where respondents indicated to what extent they personally agree or disagree with the given statements on a four-point rating scale (Wirtz et al., 2008). The score ranges from 6 to 24 with higher scores reflecting higher overcommitment (Siegrist et al., 2004). This short scale was developed from an original 29-item scale measuring different relevant aspects of this coping pattern. The short scale captures the core notion of the construct (inability to withdraw from work obligations), and resulted in predicted health outcomes of similar strength as those by the longer scale (Siegrist, 1996; Siegrist et al., 2004). Moreover, the scale exhibited the highest internal consistency in previous analyses and had an acceptable scalability (Siegrist et al., 2004). Finally, goodness of fit was appropriate in several confirmatory factor analyses (Hanson et al., 2000; Joksimovic et al., 2002; Roedel et al., 2004).

ERI assesses stressful experience at work by a 17-item questionnaire consisting of two scales measuring perceived efforts (scale effort: 6 items) and experienced or anticipated rewards (scale reward: 11 items) (Siegrist, 1996; Siegrist et al., 2004). To mirror the core theoretical notion of nonreciprocal exchange at work, a ratio of these two scales was constructed according to established recommendations (Siegrist et al., 2004).

Depressive symptom severity was assessed with the 21item Beck Depression Inventory (BDI) where scores $\geq 10$ indicate possible clinical depression (Beck et al., 1961). The BDI was developed for the assessment of depressive symptoms that correspond to the Diagnostic and Statistical Manual of Mental Disorders-IV (DSM-IV) criteria for major depressive disorder and measures a somatic and a cognitiveaffective dimension of depression (Beck et al., 1996; Kuhner et al., 2007). The BDI assesses symptoms related to sadness, feelings of guilt, perceptions of self-worth, suicidal ideation, and changes in appetite and body weight, among other characteristics.

All questionnaires have been broadly used and shown satisfactory internal consistency and validity. Questionnaires were administered on the day before blood sampling.

\section{Neuroendocrine test}

All subjects reported to the laboratory on a single study day at noon and had abstained from physical exercise, alcohol, and caffeinated beverages since the previous evening.
Combined dex/CRH tests were performed according to the protocol used in a series of previous investigations (Heuser et al., 1996; Zobel et al., 2001). After pretreatment with an oral dose of $1.5 \mathrm{mg}$ dexamethasone at $2300 \mathrm{~h}$ the evening before the test, blood samples were collected at $1500 \mathrm{~h}$, $1530 \mathrm{~h}, 1545 \mathrm{~h}, 1600 \mathrm{~h}$ and $1615 \mathrm{~h}$ through an intravenous catheter inserted between $1400 \mathrm{~h}$ and $1415 \mathrm{~h}$. The HPA system was challenged with infusion of $100 \mu \mathrm{g}$ of human CRH (Ferring, Kiel, Germany) at $1502 \mathrm{~h}$, after the first blood sample. During the whole test procedure patients rested supine but were not allowed to sleep.

The plasma cortisol and ACTH concentrations in the first sample collected at $1500 \mathrm{~h}$ reflect the suppressive effects of the dexamethasone administered the day before, whereas the other four plasma cortisol and ACTH concentrations reflect the additional effects of the $\mathrm{CRH}$ injection. We used for both, cortisol and ACTH, the five repeated consecutive blood samples as indicators of the response to the dex/CRH test. Moreover, as a further indicator, we used the maximum increase score $(\Delta \max )$ calculated as peak hormone level minus pre-test $(1500 \mathrm{~h})$ level.

\section{Neuroendocrine assays}

For determination of plasma cortisol concentrations, a commercially available radioimmunoassay (RIA) kit (ICN Biomedicals, Carson, CA) was used. The detection limit was . $3 \mathrm{ng} /$ $\mathrm{ml}$ plasma; intra- and inter-assay coefficients of variation for $20 \mathrm{ng} / \mathrm{ml}$ and $40 \mathrm{ng} / \mathrm{ml}$ were $<7 \%$. For plasma ACTH measurements an immunoradiometric assay without extraction (Nichols Institute, San Juan Capistrano, CA) was used. Detection limit of plasma ACTH concentrations was $4.0 \mathrm{pg} / \mathrm{ml}$, and the intra- and inter-assay coefficients of variation at $20 \mathrm{pg} /$ $\mathrm{ml}$ plasma were $<8 \%$.

\section{Statistical analysis}

All calculations were performed using SPSS Inc. (v11.0.1) software packages (SPSS, Chicago, IL). Data are presented as mean \pm SEM. Results were considered statistically significant at the $p \leq .05$ level. The optimal total sample size to detect an expected small to medium effect size of .10 in regression analyses with a power of .94 (5 predictors) was $n=195$. In case of missing data, cases were excluded listwise. Data were tested for normal distribution and homogeneity of variance using a Kolmogorov-Smirnov test. As neuroendocrine parameter levels and OC scores were skewed, all data were Ztransformed rendering a mean of 0 and a standard deviation of 1. Z-transformed data were used for all statistical analyses, but means and standard errors of means of original values are presented in tables and figures. We report the ANOVA effect size measures partial eta squared $\left(\eta_{p}^{2}\right)$ which refers to the proportion of the effect plus error variance that is attributable to the effect and the effect size index $f$ which indicates whether an effect is of small $(f=.10)$, medium $(f=.25)$, or large $(f=.40)$ effect size.

To assess whether $O C$ is associated with suppressive effects of dexamethasone on cortisol and ACTH pre-test levels, we calculated linear regression analyses. As dependent variable we entered the pre-test $(1500 \mathrm{~h})$ level of the respective neuroendocrine parameter. As independent variable, we entered OC while controlling for age and gender 
which were entered simultaneously. Moreover, we further controlled for depressive symptoms scores (BDI) and work stress (ERI).

To assess whether $\mathrm{OC}$ is associated with altered cortisol and $\mathrm{ACTH}$ responses to the dex/CRH test, we first calculated repeated measure analyses of covariance (ANCOVAs). As dependent variables we entered the respective neuroendocrine parameter (i.e. cortisol or ACTH) as repeated measure factor. OC was entered as continuous independent variable. To test whether a potential association between overcommitment and the neuroendocrine response to the dex/CRH test was influenced by depressive symptoms (BDI) and effortreward-imbalance (ERI) scores, we controlled for these parameters by entering them simultaneously as continuous covariates. Greenhouse-Geisser corrections were applied where appropriate. Second, we calculated regression analyses with the maximum increase score in response to $\mathrm{CRH}$ injection ( $\Delta$ max scores) of the respective neuroendocrine parameter as dependent variable. As independent variable, we entered OC while controlling for BDI and ERI which were entered simultaneously. Notably, in all of these analyses we entered $\mathrm{OC}$ as continuous variable and controlled for age and gender.

To test whether the use of hormonal contraceptives and/ or the menstrual cycle phase (i.e. luteal phase, follicular phase, lack of cycle due to menopause or ovarectomy) influences ACTH and cortisol levels or associations between $\mathrm{OC}$ and $\mathrm{ACTH}$ and cortisol levels, we entered the variables as additional dichotomous independent variables in regression and repeated ANCOVA analyses.

To illustrate our continuously calculated results we categorized the study group into tertiles based on their OC score rendering three groups of subjects with lower $(n=69)$, medium $(n=62)$, and higher $(n=69)$ OC scores.

\section{Results}

\section{Group characteristics}

The study sample consisted of 92 men and 108 women, whose sociodemographic and psychological characteristics are detailed in Table 1. As intended, participants displayed a wide range of OC scores including minimum (6) and maximum (24) OC scores with a mean of $13.25 \pm .27$ (mean \pm SEM). Information on the use or non-use of hormonal contraceptive medication $(\mathrm{HC})$ was accessible in 105 of 108 women. Of the 68 women without $\mathrm{HC}$, data on the menstrual cycle phase were available in 62 women.

\section{Overcommitment and suppressive effects of dexamethasone on cortisol and ACTH pre-test levels (negative feedback sensitivity)}

OC was not associated with ACTH $(p=.60)$ or cortisol $(p=.31) 1500 \mathrm{~h}$ pre-test levels when controlling for age (ACTH: $p=.77$; cortisol: $p=.28$ ) and gender (ACTH: $p=.67$; cortisol: $p=.06$ ). Additional controlling for depressive symptoms and ERI scores as well as for use of hormonal contraceptives or menstrual cycle phase did not significantly change these results. Notably, except HC use $\left(\beta=.29, \quad p=.001, R^{2}=.06\right)$ and age $(\beta=.19, p=.03$, $R^{2}=.03$ ), none of the other control variables were associated with higher cortisol but not ACTH ( $p$ 's $>.48)$ pretest levels.

\section{Overcommitment and the neuroendocrine response to the dex/CRH test}

ACTH: Neither ANCOVA with repeated measures (interaction time-by-OC: $p=.22)$ nor regression analyses $(\Delta \max : \beta=.05$, $p=.47$ ) were able to reveal significant associations between $\mathrm{OC}$ and $\mathrm{ACTH}$ secretion in the combined dex/CRH test (Figure $1 \mathrm{~A})$. Again, age ( $p$ 's $>.30)$ and gender ( $p$ 's $>.83$ ) were controlled.

Cortisol: ANCOVA analyses controlling for age $(p=.07)$ and gender $(p=.69)$ revealed that higher OC was associated with higher repeated measurement of cortisol secretion in the combined dex/CRH test (interaction time-by-OC: $F(1.71 /$ $\left.335.6)=4.60, p=.014, \eta_{p}^{2}=.023, f=.15\right)$ (Figure 1B). These results were confirmed by regression analyses indicating that higher OC was associated with higher cortisol $\Delta$ max scores

Table 1 Sociodemographic and psychological characteristics of the study subjects.

\begin{tabular}{|c|c|c|}
\hline & $N$ & Mean \pm SEM (observed range) \\
\hline Age [years] & 200 & $38.9 \pm .82(20-75)$ \\
\hline Gender $[\mathrm{N}]$ & 200 & 92 men, 108 women \\
\hline $\begin{array}{l}\text { Women with and without intake of hormonal } \\
\text { contraceptive }(\mathrm{HC}) \text { medication }[\mathrm{N}]\end{array}$ & $37 / 68$ & \\
\hline $\begin{array}{l}\text { Women without } \mathrm{HC} \text { in the follicular phase of } \\
\text { the menstrual cycle [N] }\end{array}$ & 28 & \\
\hline $\begin{array}{l}\text { Women without } \mathrm{HC} \text { in the luteal phase of the } \\
\text { menstrual cycle }[\mathrm{N}]\end{array}$ & 18 & \\
\hline $\begin{array}{l}\text { Women without } \mathrm{HC} \text { and without menstrual } \\
\text { cycle due to menopause or ovarectomy [N] }\end{array}$ & 16 & \\
\hline Overcommitment [OC score] & 200 & $13.25 \pm .27(6-24)$ \\
\hline Depressive symptoms [BDI score] & 196 & $4.48 \pm .33(0-26)$ \\
\hline Work Stress [ERI score] & 198 & $.54 \pm .02(.20-2.01)$ \\
\hline High school degree ("Abitur/Fachabitur") [\%] & 121 & 60.5 \\
\hline Full- or part-time job [\%] & 161 & 80.5 \\
\hline Weekly work time [hours] & 188 & $39.84 \pm .83(5-70)$ \\
\hline
\end{tabular}

$\mathrm{N}$ : valid cases, follicular phase: days 1-14; luteal phase: days 15-28. 

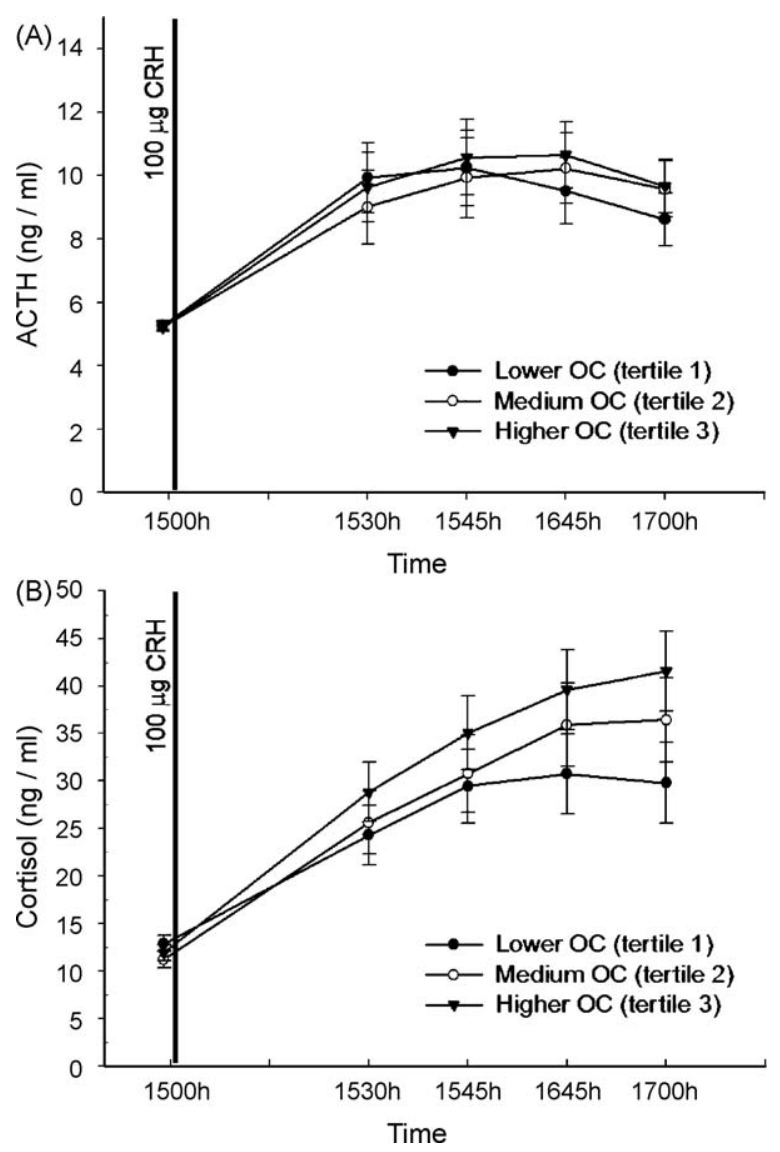

Figure 1 ACTH (Panel A) and Cortisol (Panel B) responses to the combined dex/CRH-Test in three groups of subjects with lower, medium, and higher overcommitment (OC) scores. Values are means \pm SEM. ANCOVA analyses controlling for age and gender revealed that higher $O C$ (entered as continous variable) was associated with higher repeated measurement of cortisol secretion in response to the combined dex/CRH test $(F(1.71 /$ $335.9)=4.59, p=.014, f=.15)$ (Panel 1B). In contrast, there was no association between $\mathrm{OC}$ and ACTH secretion $(p=.22)$ (Panel A).

$\left(\beta=.15, p=.029, R^{2}=.02\right)$ while controlling for age $(\beta=.20$, $\left.p=.006, R^{2}=.04\right)$ and gender $(p=.10)$.

\section{Influence of depressive symptoms and effort- reward imbalance on the association between overcommitment and the neuroendocrine response to the dex/CRH test}

We additionally entered BDI and ERI scores as further covariates in previous ANCOVAs and as further predictors in previous regression analyses.

ACTH: BDI and ERI were not significantly associated with repeated ACTH measures ( $p$ 's $>.31$ ) or with ACTH (max scores ( $p$ 's $>.37)$. Moreover, they did not significantly change the non-significant associations between $O C$ and these ACTH measures.

Cortisol: Neither BDI nor ERI were significantly associated with repeated cortisol measures ( $p$ 's $>.54$ ), and they were not significant predictors of cortisol $\Delta \max$ scores ( $p$ 's $>.47$ ).
Controlling for BDI and ERI did not significantly change the association between $\mathrm{OC}$ and repeated cortisol measures (interaction time-by-OC: $F(1.70 / 320.4)=3.09, p=.030, \eta_{p}^{2}=.020$, $f=.14)$ or between OC and cortisol $\Delta \max$ scores $(\beta=.16$, $p=.05, R^{2}=.02$ ).

\section{Influence of hormonal contraceptive use and the female menstrual cycle phase on the neuroendocrine response to the dex/CRH test}

We additionally entered four dichotomous variables coding the use of hormonal contraceptives $(\mathrm{HC})$, the luteal phase of the menstrual cycle, the follicular phase of the menstrual cycle, and the lack of a menstrual cycle (due to menopause/ ovarectomy) as covariates instead of the gender variable.

ACTH: HC use and menstrual cycle phase variables (i.e. luteal phase, follicular phase, lack of cycle due to menopause or ovarectomy) were not significantly associated with repeated ACTH levels ( $p$ 's $>.49$ ) or ACTH (max scores ( $p$ 's $>.09$ ), nor did they significantly change the non-significant association between OC and ACTH measures $(p=.85)$.

Cortisol: Whereas menstrual cycle phase was not significantly associated with repeated cortisol levels ( $p$ 's $>.15$ ), there was a significant association between $\mathrm{HC}$ use and repeated cortisol levels $(F(1.80 / 320.6)=6.65 p=.002$, $\left.\eta_{p}^{2}=.036, f=.19\right)$. Controlling for $\mathrm{HC}$ use and menstrual cycle phase lowered the association between $O C$ and repeated cortisol measures to marginal significance (interaction timeby-OC: $\left.F(1.80 / 320.6)=3.02, p=.053, \eta_{p}^{2}=.017, f=.13\right)$. In terms of cortisol $\Delta$ max scores only follicular phase was associated with higher cortisol $\Delta \max$ scores $(\beta=.24, p=.002$, $\left.R^{2}=.05\right)$. Controlling for $\mathrm{HC}$ use and menstrual cycle phase lowered the association between $\mathrm{OC}$ and cortisol $\Delta$ max scores to marginal significance $\left(\beta=.16, p=.062, R^{2}=.02\right)$.

\section{Discussion}

To assess potential associations between $\mathrm{OC}$ and alterations in HPA system function we measured ACTH and cortisol release in response to the combined dex/CRH test while controlling for potential confounders in a sizable number of healthy middle-aged men and women. The main finding of our study was that $\mathrm{OC}$ is independently associated with higher increases in cortisol but not ACTH levels after a physiological $\mathrm{CRH}$ challenge following pre-treatment with dexamethasone while there were no associations between $\mathrm{OC}$ and secretion of ACTH and cortisol after the exclusive administration of dexamethasone. The finding of elevated $\mathrm{CRH}$-induced cortisol increases with $\mathrm{OC}$ was of small to medium effect size.

Our findings suggest an association between OC and HPA axis dysregulation. Higher $\mathrm{OC}$ levels were accompanied by increased cortisol secretion in the combined dex/CRH test while ACTH secretion was independent of OC levels in this paradigm. This divergence may be due to the differential stress signal processing at the various levels of the HPA system, e.g. hippocampus, hypothalamus, pituitary, adrenal cortex (Holsboer, 2000). Furthermore, the negative feedback sensitivity of the HPA system does not seem to be affected by $\mathrm{OC}$, as indicated by the independence of the dexamethasone-suppressed ACTH and cortisol secretion ( $1500 \mathrm{~h}$ data) from OC scores.

How do our findings relate to the literature on HPA system functioning, and HPA axis stress reactivity with OC? First, 
with respect to HPA system functioning, our finding of feedback sensitivity unaffected by $\mathrm{OC}$ levels as indicated by the $1500 \mathrm{~h} \mathrm{ACTH}$ and cortisol plasma concentrations before CRH injection is in line with a recent study by Bellingrath et al. (2008). In that study, the authors assessed OC and the cortisol awakening response as well as a circadian salivary cortisol profile after pre-medication with $.25 \mathrm{mg}$ dexamethasone the night before in 120 middle-aged school teachers. They did not report associations between OC and alterations in HPA axis feedback sensitivity. However, to the best of our knowledge, our study is the first to assess the effects of a combined dex/CRH challenge on HPA axis regulation, which is suggested to be more sensitive to revealing even subtle alterations of HPA axis function, which are not obvious when only investigating the feedback loop of the HPA system.

Our findings on increasing cortisol secretion with elevating OC levels together with unaffected ACTH secretion subsequent to $\mathrm{CRH}$ injection are in line with some previous observations on elevated basal HPA axis activity, both over the work day and in response to awakening (Steptoe et al., 2004; Hurwitz-Eller et al., 2006) although not unequivocally (Bellingrath et al., 2008). More precisely, our findings may explain elevated cortisol levels with $O C$ as they suggest heightened sensitivity of the adrenal cortex to the incoming ACTH signal as one potential underlying mechanism. In addition, ACTH independent glucocorticoid regulation may play a role (Engeland and Arnhold, 2005; Bornstein et al., 2008).

Second, when integrating our findings into the literature on stress reactivity of the HPA axis with OC, and in particular the previously observed blunted ACTH and cortisol reaction to an acute psychological stress situation (Bellingrath and Kudielka, 2008; Wirtz et al., 2008), it may be suggested that stress-induced $\mathrm{CRH}$ release is blunted with increasing $\mathrm{OC}$. Thus, even a hyperreactivity of the adrenal cortex with $O C$ may not sufficiently counteract and thereby normalize neuroendocrine stress reactivity resulting in the observed reduction of ACTH and cortisol secretion. Notably, the temperament-related personality factors novelty seeking and harm avoidance have been shown to be consistently associated with either higher or lower cortisol reactivity in the dex/CRH test and in response to psychosocial stress (Tyrka et al., 2007, 2008). In contrast, OC shows a divergent pattern with lower cortisol stress reactivity (Bellingrath and Kudielka, 2008; Wirtz et al., 2008) and higher cortisol reactivity in the dex/CRH test as observed in the present study. A speculative explanation for this discrepancy may relate to the idea of a hyperreactive adrenal cortex with $\mathrm{OC}$ as a consequence of adaptive mechanisms in reaction to frequent OC-triggered stress experiences. Thus, in line with the concept of allostatic load (McEwen, 1998) a hyperreactive adrenal cortex with $\mathrm{OC}$ may be an adaptive reaction to an "exhausted" stress responsive system and thus may cause the observed reactivity difference in response to stress and in response to the dex/CRH test. In contrast, novelty seeking and harm avoidance within subclinical ranges as observed in the studies by Tyrka et al. $(2007,2008)$ may not be associated with adaptive alterations in HPA functioning.

From a clinical perspective, similarities and differences between $\mathrm{OC}$ and depression concerning HPA system regulation are of interest. $O C$ is associated with an elevated risk of depression (Dragano et al., 2008), and these two conditions, OC (Siegrist et al., 1990; Joksimovic et al., 1999; Kuper et al.,
2002), and depression (Musselman et al., 1998; Parissis et al., 2007; Van der Kooy et al., 2007), were found to increase the risk of CHD. Both of these risk factors of CHD show elevated cortisol secretion in the combined dex/CRH test, suggesting an HPA system dysregulation (Zobel et al., 2001; Hatzinger et al., 2002; Ising et al., 2005). However, while we were unable to observe a correlation between ACTH secretion and OC levels, some, but not all, studies suggested elevated pituitary reactivity, as evidenced by increased ACTH release following $\mathrm{CRH}$ injection in the combined dex/CRH test with depression (Heuser et al., 1994; Schule et al., 2003). Notably, as the observed ACTH levels in our study are in the lower detection range of the assay, we cannot rule out that potential measurement errors may contribute to our non-significant ACTH findings. Whereas we and others have so far been unable to find OC-related alterations in negative feedback regulation of the HPA system (Bellingrath et al., 2008), non-suppression of hormonal secretion subsequently to the administration of dexamethasone is often, but not always, described in depression (Arana et al., 1985; Ehlert et al., 2001). Taken together, OC and depression might share a common biological mechanism in terms of HPA system dysregulation.

Interestingly, in a sample of depressed women with longterm sick leave who attributed their disability to job-related stress, Rydmark et al. recently reported blunted ACTH and cortisol secretion in the dex/CRH test (Rydmark et al., 2006). As this finding was contrary to the authors' expectations they attributed the lower instead of higher neuroendocrine reactivity in part to the fact that the vast majority of their participants had first-episode depression. Moreover, they interpreted that the dex/CRH results observed in their job-stress-induced depressive women are indicative of hypoactive central stress-axis circuitry similar to what has been described for atypical depression (Rydmark et al., 2006). In our study in healthy non-depressive men and women neither depressive symptom severity nor job stress was associated with neuroendocrine reactivity in the dex/CRH test. It is therefore possible that the depression and ERI levels observed in our study were too low and homogeneous and thereby too "normal" to be able to influence ACTH and cortisol responses in the dex/CRH test. Future studies should investigate whether our $\mathrm{OC}$ findings persist in patients with major depression and in subjects with high job strain (as indicated by ERI scores $\geq 1$ ).

How might our HPA findings in terms of OC relate to biological pathways that underlie the elevated CHD risk with OC? In line with Appels' model of the association of vital exhaustion with acute coronary syndromes (Appels, 1999), OC might lead to prolonged stress and overexertion. Indeed, $\mathrm{OC}$ is positively associated with vital exhaustion (Preckel et al., 2005; Wirtz et al., 2008). The HPA system dysregulation may induce the reported elevated basal cortisol levels with OC (Steptoe et al., 2004; Hurwitz-Eller et al., 2006), which according to Appels may cause an adaptive decreased immuno-competence, resulting in reactivation of systemic infections and release of inflammatory cytokines (Appels, 1999). HPA system dysregulation might be the underlying pathomechanism of reduced immuno-competence and endogenous inflammation, triggering acute coronary syndromes and CHD risk (Appels, 1999; Ridker et al., 2000a,b) in overcommitted individuals, as well as of the development of symptoms of depression (Dantzer et al., 2008). 
There are several positive aspects to our study that are worthy of mention. First, in order to test HPA system functioning in $\mathrm{OC}$ we used the hitherto most sensitive tool for detection of HPA system dysregulation which is robust against many confounding influences (Heuser et al., 1994; Kunzel et al., 2003). Second, we recruited a sizable number of healthy middle-aged men and women in order to guarantee a sufficient statistical power, which allows potential and well-known confounders to be controlled for and enables the generalization of our findings to both sexes. Third, we used a statistical approach that allowed the assessment of linear associations between $O C$ measured as a continuous variable and changes in cortisol and ACTH plasma levels in the combined dex/CRH test in the study group. Notably, we refrained in this regard from reducing the repeatedly measured hormonal response to the test to a single measure index, but rather included all measurements while accounting for repeated measures. However, limitations of our study also need to be addressed. First, our data are cross-sectional, so that causal interpretations of the observed associations, although plausible, remain speculative. Second, we excluded psychiatric diseases including major depressive disorder. Therefore, it remains to be elucidated whether our $O C$ findings persist in patients with major depression. Similarly, the work strain levels of our subjects were low and homogeneous (as indicated by the low ERI-SEM). Therefore, the variability of ERI-scores may have been too low to detect potential associations with neuroendocrine changes suggested by a previous study (Rydmark et al., 2006). Third, the clinical implications of our findings still need to be elucidated.

In conclusion, we found a positive correlation between $\mathrm{OC}$ and cortisol secretion in the dex/CRH test in healthy volunteers. Future research is needed to determine the clinical implications of our findings and the underlying biological mechanisms in the interface between OC, depression, and CHD risk.

\section{Role of funding source}

Funding for this study was provided by the Department of Psychiatry, University of Bonn.

\section{Conflict of interest}

None declared.

\section{Acknowledgements}

None.

\section{References}

Appels, A., 1999. Inflammation and the mental state before an acute coronary event. Ann. Med. 31 (Suppl. 1), 41-44.

Arana, G.W., Baldessarini, R.J., Ornsteen, M., 1985. The dexamethasone suppression test for diagnosis and prognosis in psychiatry. Commentary and review. Arch. Gen. Psychiatry 42, 1193-1204.

Beck, A.T., Ward, C.H., Mendelson, M., Mock, J., Erbaugh, J., 1961. An inventory of measuring depression. Arch. Gen. Psychiatry 45, 561-571.
Beck, A.T., Steer, R.A., Ball, R., Ranieri, W., 1996. Comparison of beck depression inventories -IA and -II in psychiatric outpatients. J. Pers. Assess. 67, 588-597.

Bellingrath, S., Kudielka, B.M., 2008. Effort-reward-imbalance and overcommitment are associated with hypothalamus-pituitaryadrenal (HPA) axis responses to acute psychosocial stress in healthy working schoolteachers. Psychoneuroendocrinology 33, 1335-1343.

Bellingrath, S., Weigl, T., Kudielka, B.M., 2008. Cortisol dysregulation in school teachers in relation to burnout, vital exhaustion, and effort-reward-imbalance. Biol. Psychol. 78, 104-113.

Bornstein, S.R., Engeland, W.C., Ehrhart-Bornstein, M., Herman, J.P., 2008. Dissociation of ACTH and glucocorticoids. Trends Endocrinol. Metab. 19 (5), 175-180.

Dantzer, R., O’Connor, J.C., Freund, G.G., Johnson, R.W., Kelley, K.W., 2008. From inflammation to sickness and depression: when the immune system subjugates the brain. Nat. Rev. Neurosci. 9, 46-56.

Dragano, N., He, Y., Moebus, S., Jockel, K.H., Erbel, R., Siegrist, J., 2008. Two models of job stress and depressive symptoms. Results from a population-based study. Soc. Psychiatry Psychiatry Epidemiol. 43, 72-78.

Ehlert, U., Gaab, J., Heinrichs, M., 2001. Psychoneuroendocrinological contributions to the etiology of depression, posttraumatic stress disorder, and stress-related bodily disorders: the role of the hypothalamus-pituitary-adrenal axis. Biol. Psychol. 57, 141-152.

Engeland, W.C., Arnhold, M.M., 2005. Neural circuitry in the regulation of adrenal corticosterone rhythmicity. Endocrine 28 (3), $325-332$.

Girod, J.P., Brotman, D.J., 2004. Does altered glucocorticoid homeostasis increase cardiovascular risk? Cardiovasc. Res. 64, 217-226.

Hanson, E.K., Schaufeli, W., Vrijkotte, T., Plomp, N.H., Godaert, G.L., 2000. The validity and reliability of the Dutch effort-reward imbalance questionnaire. J. Occup. Health Psychol. 5, 142-155.

Hatzinger, M., Hemmeter, U.M., Baumann, K., Brand, S., HolsboerTrachsler, E., 2002. The combined DEX-CRH test in treatment course and long-term outcome of major depression. J. Psychiatry Res. 36, 287-297.

Heuser, I., Yassouridis, A., Holsboer, F., 1994. The combined dexamethasone/CRH test: a refined laboratory test for psychiatric disorders. J. Psychiatry Res. 28, 341-356.

Heuser, I.J., Schweiger, U., Gotthardt, U., Schmider, J., Lammers, C.H., Dettling, M., Yassouridis, A., Holsboer, F., 1996. Pituitaryadrenal-system regulation and psychopathology during amitriptyline treatment in elderly depressed patients and normal comparison subjects. Am. J. Psychiatry 153, 93-99.

Holsboer, F., 2000. The corticosteroid receptor hypothesis of depression. Neuropsychopharmacology 23, 477-501.

Holsboer, F., von Bardeleben, U., Wiedemann, K., Muller, O.A., Stalla, G.K., 1987. Serial assessment of corticotropin-releasing hormone response after dexamethasone in depression. Implications for pathophysiology of DST nonsuppression. Biol. Psychiatry 22, 228-234.

Hurwitz-Eller, N., Netterstrom, B., Hansen, A.M., 2006. Psychosocial factors at home and at work and levels of salivary cortisol. Biol. Psychol. 73, 280-287.

Ising, M., Kunzel, H.E., Binder, E.B., Nickel, T., Modell, S., Holsboer, F., 2005. The combined dexamethasone/CRH test as a potential surrogate marker in depression. Prog. Neuropsychopharmacol. Biol. Psychiatry 29, 1085-1093.

Joksimovic, L., Siegrist, J., Meyer-Hammer, M., Peter, R., Franke, B., Klimek, W.J., Heintzen, M.P., Strauer, B.E., 1999. Overcommitment predicts restenosis after coronary angioplasty in cardiac patients. Int. J. Behav. Med. 6, 356-369.

Joksimovic, L., Starke, D., Von Dem Knesebeck, O., Siegrist, J., 2002. Perceived work stress, overcommitment, and self-reported musculoskeletal pain: a cross-sectional investigation. Int. J. Behav. Med. 9, 122-138. 
Kuhner, C., Burger, C., Keller, F., Hautzinger, M., 2007. Reliability and validity of the revised beck depression inventory (BDI-II), results from German samples. Nervenarzt 78, 651-656.

Kunzel, H.E., Binder, E.B., Nickel, T., Ising, M., Fuchs, B., Majer, M., Pfennig, A., Ernst, G., Kern, N., Schmid, D.A., Uhr, M., Holsboer, F., Modell, S., 2003. Pharmacological and nonpharmacological factors influencing hypothalamic-pituitary-adrenocortical axis reactivity in acutely depressed psychiatric in-patients, measured by the Dex-CRH test. Neuropsychopharmacology 28, 2169-2178.

Kuper, H., Singh-Manoux, A., Siegrist, J., Marmot, M., 2002. When reciprocity fails: effort-reward imbalance in relation to coronary heart disease and health functioning within the Whitehall II study. Occup. Environ. Med. 59, 777-784.

Lett, H.S., Blumenthal, J.A., Babyak, M.A., Sherwood, A., Strauman, T., Robins, C., Newman, M.F., 2004. Depression as a risk factor for coronary artery disease: evidence, mechanisms, and treatment. Psychosom. Med. 66, 305-315.

McEwen, B.S., 1998. Protective and damaging effects of stress mediators. N. Engl. J. Med. 338 (3), 171-179.

Musselman, D.L., Evans, D.L., Nemeroff, C.B., 1998. The relationship of depression to cardiovascular disease: epidemiology, biology, and treatment. Arch. Gen. Psychiatry 55, 580-592.

Parissis, J.T., Fountoulaki, K., Filippatos, G., Adamopoulos, S., Paraskevaidis, I., Kremastinos, D., 2007. Depression in coronary artery disease: novel pathophysiologic mechanisms and therapeutic implications. Int. J. Cardiol. 116, 153-160.

Preckel, D., von Kanel, R., Kudielka, B.M., Fischer, J.E., 2005. Overcommitment to work is associated with vital exhaustion. Int. Arch. Occup. Environ. Health 78, 117-122.

Ridker, P.M., Rifai, N., Pfeffer, M., Sacks, F., Lepage, S., Braunwald, E., 2000a. Elevation of tumor necrosis factor-alpha and increased risk of recurrent coronary events after myocardial infarction. Circulation 101, 2149-2153.

Ridker, P.M., Rifai, N., Stampfer, M.J., Hennekens, C.H., 2000b. Plasma concentration of interleukin- 6 and the risk of future myocardial infarction among apparently healthy men. Circulation 101, 1767-1772.

Roedel, A., Siegrist, J., Hessel, A., Braehler, E., 2004. Psychometric test of the questionnaire measuring effort-reward imbalance at work in a representative German sample. Zeitschrift für Differentielle und Diagnostische Psychologie 24, 227-238.

Rydmark, I., Wahlberg, K., Ghatan, P.H., Modell, S., Nygren, A., Ingvar, M., Asberg, M., Heilig, M., 2006. Neuroendocrine, cognitive and structural imaging characteristics of women on longterm sickleave with job stress-induced depression. Biol. Psychiatry 60 , 867-873.

Schule, C., Baghai, T., Zwanzger, P., Ella, R., Eser, D., Padberg, F., Moller, H.J., Rupprecht, R., 2003. Attenuation of hypothalamicpituitary-adrenocortical hyperactivity in depressed patients by mirtazapine. Psychopharmacology (Berl.) 166, 271-275.

Siegrist, J., 1996. Adverse health effects of high-effort/low-reward conditions. J. Occup. Health Psychol. 1, 27-41.

Siegrist, J., Peter, R., Junge, A., Cremer, P., Seidel, D., 1990. Low status control, high effort at work and ischemic heart disease: prospective evidence from blue-collar men. Soc. Sci. Med. 31, 1127-1134.

Siegrist, J., Starke, D., Chandola, T., Godin, I., Marmot, M., Niedhammer, I., Peter, R., 2004. The measurement of effort-reward imbalance at work: European comparisons. Soc. Sci. Med. 58, 1483-1499.

Steptoe, A., Siegrist, J., Kirschbaum, C., Marmot, M., 2004. Effortreward imbalance, overcommitment, and measures of cortisol and blood pressure over the working day. Psychosom. Med. 66, 323-329.

Tyrka, A.R., Wier, L.M., Anderson, G.M., Wilkinson, C.W., Price, L.H., Carpenter, L.L., 2007. Temperament and response to the Trier Social Stress Test. Acta Psychiatry Scand. 115, 395402.

Tyrka, A.R., Wier, L.M., Price, L.H., Rikhye, K., Ross, N.S., Anderson, G.M., Wilkinson, C.W., Carpenter, L., 2008. Horm. Behav. 53, 518-525.

Van der Kooy, K., van Hout, H., Marwijk, H., Marten, H., Stehouwer, C., Beekman, A., 2007. Depression and the risk for cardiovascular diseases: systematic review and meta analysis. Int. J. Geriatr. Psychiatry 22, 613-626.

Wirtz, P.H., Siegrist, J., Rimmele, U., Ehlert, U., 2008. Higher overcommitment to work is associated with lower norepinephrine secretion before and after acute psychosocial stress in men. Psychoneuroendocrinology 33, 92-99.

Wittchen, H.U., Perkonigg, A., 1997. DIA-X-Screening Verfahren: Fragebogen DIA-X-SSQ. Swets \& Zeitlinger, Frankfurt.

Wittchen, H.U., Pfister, H., 1997. DIA-X Manual: Instruktionsmanual zur Durchführung von DIA-X (M-CIDI) Interviews. Swets Test Services, Frankfurt.

Zobel, A.W., Nickel, T., Sonntag, A., Uhr, M., Holsboer, F., Ising, M., 2001. Cortisol response in the combined dexamethasone/CRH test as predictor of relapse in patients with remitted depression. a prospective study. J. Psychiatry Res. 35, 83-94. 